\title{
Analysis and simulation of the structure of nanoparticles that undergo a surface-driven structural transformation
}

\author{
Benjamin Gilbert ${ }^{\text {a) }}$, Hengzhong Zhang, Feng Huang \\ Department of Earth and Planetary Sciences, University of California - Berkeley, CA 94720.
}

Yang Ren, Daniel Haskel, J. C. Lang and George Srajer

Advanced Photon Source, Argonne National Laboratory, Argonne, IL 60439

Astrid Jürgensen

Canadian Synchrotron Radiation Facility, UW-Madison SRC, Stoughton, WI 53589-3097

Glenn A. Waychunas, and Jillian F. Banfield ${ }^{\text {a) }}$

Lawrence Berkeley National Lab, One Cyclotron Road, Berkeley, CA 94720

\section{ABSTRACT}

A room temperature solid state structural transformation was observed in $3 \mathbf{~ n m}$ ZnS nanoparticles in methanol following the addition of water (Zhang et al., Nature, 424, 1025, 2003). Experimental wide angle x-ray scattering (WAXS), x-ray absorption near edge structure (XANES) and extended $x$-ray absorption fine structure (EXAFS) spectroscopy measurements show a large increase in crystallinity associated with water addition, in agreement with molecular dynamics (MD) predictions. Here we perform first-shell EXAFS and pair distribution function (PDF) analysis and whole-nanoparticle calculations of WAXS, EXAFS and XANES to compare structural data with the MD predictions. The predicted WAXS patterns give excellent agreement with data, while the predicted EXAFS and XANES spectra give poor agreement. Relative to WAXS, XANES and EXAFS spectra contain additional structural information related to the distribution of disorder. The discrepancy between the x-ray diffraction and x-ray absorption results indicates that structural disorder is partitioned between interior and surface regions more strongly than predicted in the MD simulations. 


\section{INTRODUCTION}

The importance of nanoparticles in current research and technology is growing because basic materials properties can be affected by small size [1,2]. Two principal effects have been demonstrated. In semiconducting nanoparticles, when particle size approaches, or is smaller than, the excitonic Bohr radius, confinement causes an opening of the band gap. In addition, when the surface area is large and surface energy becomes a significant contributor to the total energy, modifications or inversions in polymorph stability have been observed in several systems $[3,4]$. These considerations imply that phase stability may be sensitive to the surface environment. Because different polymorphs often posses substantially altered electronic structures [5], environment-driven transformations may dramatically modify nanomaterial properties.

We have confirmed experimentally that the $\mathrm{ZnS}$ nanoparticle structure changes in response to ligand interactions $[6,7]$. At room temperature and pressure, macroscopic $\mathrm{ZnS}$ is a wide bandgap semiconductor that occurs as the cubic (zinc blende) sphalerite structure in which $\mathrm{Zn}$ and $\mathrm{S}$ are tetrahedrally coordinated. At high temperature, $\mathrm{ZnS}$ adopts the hexagonal wurtzite structure. However, wurtzite is encountered at relatively low temperature in nanocrystalline $\mathrm{ZnS}$ [Error! Reference source not found.], probably because of a surface energy-controlled change in phase stability. $3 \mathrm{~nm} \mathrm{ZnS}$ nanoparticles prepared in anhydrous methanol are highly distorted. Water binding, methanol desorption [6], and aggregation [7] can lead to structural changes at room temperature, reversible in the last two cases. The observation of reversible transitions establishes that $\mathrm{ZnS}$ nanoparticles are not kinetically trapped in a metastable structure.

Analysis of the relationships between phase stability and the surface environment requires in situ methods that can probe structure. Extended x-ray absorption fine structure (EXAFS) and x-ray absorption near-edge structure (XANES) spectroscopies 
offer element-specific sensitivity to coordination, oxidation state and near-neighbour geometry. Many groups have studied the structure and lattice dynamics of nanoparticles with EXAFS $[9,10,11,12,13,14]$. For example, EXAFS work on carefully characterized nanoparticle systems has revealed structural variations due to synthesis conditions and the nature of the surface ligand. With careful choice of system, the technique has been made quite surface sensitive. However, EXAFS analyses are generally limited to the first-shell in nanoparticles, and structural disorder can complicate bond-length analysis $[15,16]$. Wide-angle $x$-ray scattering (WAXS) with high-energy x-rays gives diffraction data to high momentum transfer (here, $Q_{\max }=22 \AA^{-1}$ ), with accurate solvent background subtraction. Fourier analysis of the WAXS data provides the real-space pair distribution function (PDF). PDF analysis is not yet widely used in nanoscale systems, yet provides a view of longer-range correlations [17,18]. Applied to powder samples, both these experimental methods yield average structural information that do not precisely reveal the specific modifications that arise due to small particle size. Hence, complementary theoretical structural studies are of value.

Molecular modelling is a particularly valuable tool for investigating the surface and interior structure of nanocrystalline materials. No experimental approach can presently determine the surface structure of nanoparticles, and the only information on the surface geometries comes from theory $[19,20,21]$. These details are important for determining surface energy, and hence phase stability. While the concept of excess quantities (such as surface free energy) offers a convenient continuum thermodynamical framework for prediction of the phase stability of small particles, it is incomplete, neglecting specific atomic geometry and chemical interactions. Nanoparticles typically are larger than can be conveniently handed by ab initio quantum chemistry methods. Hence, classical molecular dynamics simulations (MD) are an effective compromise between speed and accuracy, and have been used to predict surface reconstruction, surface energy, and polymorph stability in both bulk and nanoscale solids [22,23,24,25]. 
In this paper we explore the structural states of $\mathrm{ZnS}$ nanoparticles that undergo a surface environment-driven structural transformation by predicting spectra using MD simulations and comparing them with data from three in situ characterization methods. This work used the published ZnS MD potentials of Wright [22]. These were chosen because they were able to successfully describe bulk $\mathrm{ZnS}$ properties, including the structure of terminated bulk $\mathrm{ZnS}[23,24]$. We extracted model-independent physical parameters from the EXAFS data and directly compared them to the MD nanoparticle structure simulations. In addition, we used the MD structures as starting points for whole-nanoparticle calculations of experimental spectra. The x-ray absorption calculations were performed with the multiple-scattering code FEFF 8.2 [26,27], which has been widely used for EXAFS and XANES analysis of bulk and nanoscale systems $[31,32,33]$. We conclude that the approach involving integration of whole nanoparticle predictions and simulations can be applied to other small-scale systems to improve understanding of structural heterogeneity.

\section{METHODS}

\section{A. Experimental methods}

\section{ZnS nanoparticle synthesis and handling}

In order to study the effect of surface environment on nanoparticle structure, we synthesized $3 \mathrm{~nm}$ sphalerite structure $\mathrm{ZnS}$ in anhydrous methanol by drop-wise addition of $1 \mathrm{M} \mathrm{ZnCl}_{2}$ to $1 \mathrm{M} \mathrm{Na}_{2} \mathrm{~S}$ under a $\mathrm{N}_{2}$ atmosphere at room temperature. The details of the synthesis method and initial characterization results were reported previously [6]. After synthesis, two $2 \mathrm{~mL}$ aliquots were taken from the suspension, and $100 \mu \mathrm{L} \mathrm{H}_{2} \mathrm{O}$ was added to one of these. Both were sonicated for 30 minutes, and stored overnight. In situ WAXS, XANES and EXAFS data obtained from portions of these samples indicate a dramatic increase in crystallinity following water addition. 
A portion of the as-synthesized $\mathrm{ZnS}$ nanoparticle suspension in methanol was rapidly dried, by vacuum pumping down to $10^{-6}$ Torr at room temperature, producing a fine powder. XRD analysis showed no evidence for structural change associated with rapid drying [6]. The nanoparticles were handled in a nitrogen atmosphere and analyzed by WAXS and EXAFS.

In a separate experiment, $3 \mathrm{~nm} \mathrm{ZnS}$ nanoparticles were rapidly vacuum dried, as above. A portion of the dry powder was transferred to another vacuum chamber for methanol desorption. A quadrupole mass spectrometer was used to observe the partial pressures of water and methanol during sample heating, above a base pressure of $<1 \mathrm{x}$ $10^{-8}$ Torr. After observing substantial methanol evolution at $50{ }^{\circ} \mathrm{C}$, the sample was held at this temperature for 24 hours. At the end of this period, the partial pressure of methanol had fallen back to its initial value. Both degassed and non-degassed powders were pressed into indium foil (in a dry nitrogen atmosphere) and loaded into another UHV chamber for S K edge EXAFS spectroscopy.

Pure synthetic bulk wurtzite (hexagonal) and sphalerite (cubic) $\mathrm{ZnS}$ were used as reference materials for all EXAFS experiments. The former was synthesized by high temperature treatment of sphalerite, and both were shown to be pure phase by XRD.

\section{XANES and EXAFS spectroscopy}

Zn K edge x-ray absorption spectroscopy was performed on beamline 4-ID-D at the Advanced Photon Source (APS), Argonne, in transmission mode. The photon energy was calibrated before the experiment with $\mathrm{Zn}$ foil. Reference powders were brushed onto sticky tape, and layered to achieve an edge jump of approximately unity. Nanoparticle samples, dry or as a paste in methanol, were mounted in a sample holder between Kapton ${ }^{\circledR}$ membranes, and compression sealed with a Teflon gasket. Samples 
were re-prepared until the correct edge jump was obtained. EXAFS data was collected at room temperature, to $k=18 \AA^{-1}$, with constant $k$-step, and 1-3 scans were averaged. A finer energy step close to the edge was used during acquisition of XANES data. S K edge EXAFS were acquired on the DCM beamline at the University of Wisconsin Madison Synchrotron Radiation Center. Total electron yield (TEY) and fluorescent (FY) yield signals were collected from samples pressed into indium foil. Only the TEY data were analyzed due to better signal quality. Further details on the analysis can be found in Ref. 6.

\section{Wide-angle $X$-ray scattering}

WAXS patterns of ZnS nanoparticles, dry, or as suspensions in methanol, were acquired on beamline 11-ID-C at the APS at $115.2368 \mathrm{keV}(\lambda=0.10759 \AA)$. Wet samples were prepared to ensure a constant $\mathrm{ZnS}$ density, using Kapton ${ }^{\circledR}$ membranes as entrance and exit windows, diameter $7 \mathrm{~mm}$, beam path $6.5 \mathrm{~mm}$. The WAXS pattern of a

methanol blank sample (for nanoparticle suspensions) or Kapton ${ }^{\circledR}$ (for nanoparticle powders) was used for background subtraction. All patterns were acquired over a range of diffraction vector $Q=0.3-35 \AA^{-1} .2-5$ scans were averaged.

\section{B. Theoretical Calculations and Data Analysis}

\section{Molecular dynamics simulations}

Details of the MD simulations may be found elsewhere [6, 25]. Briefly, $\mathrm{ZnS}$ was described using a shell model with a Buckingham form of the pair-wise interatomic potential, plus an angle-bending form of the three-body interaction between nearest neighbor S-Zn-S atoms [22]. The short range Zn-O and S-H interactions also assume a Buckingham potential function, obtained from fits to first principles calculations for the aqueous $\mathrm{Zn}$ [28] and gas phase $\mathrm{H}_{2} \mathrm{~S}$ [29], respectively. The shell model of water showed 
best compatibility with the $\mathrm{ZnS}$ potentials [30]. Methanol is a weakly polar solvent compared to water. Hence the interactions between methanol and $\mathrm{ZnS}$ are expected to be much weaker than between water and $\mathrm{ZnS}$. We therefore performed MD simulations of $\mathrm{ZnS}$ nanoparticles in vacuum for comparison with experimental data in methanol. A spherical model ZnS nanoparticle was prepared with the ideal $\mathrm{ZnS}$ sphalerite structure. Surface atoms were removed to achieve charge neutrality, choosing highly underbonded atoms preferentially. The molecular dynamics simulations were run to minimize the total energy of the resulting model nanoparticle in a canonical assemble at $300 \mathrm{~K}$ using a Nose-Hoover algorithm, or (optionally, at large times) a Gaussian algorithm, with a time step of 0.5 fs for no less than 100 ps.

Fig. 1 shows the energetic state diagram of the MD simulations. The initial structure was a 1, 2, 2.5, 3 or $5 \mathrm{~nm}$ diameter spherical model nanoparticle sphalerite or wurtzite. The structure was dynamically relaxed either in vacuum, or with 1,3 or 5 molecules of water $/ \mathrm{nm}^{2}$ on the surface. In all simulated XRD patterns, structural relaxation caused loss of peak intensity relative to the initial (unrelaxed) structure. The simulated patterns for relaxed $3 \mathrm{~nm} \mathrm{ZnS}$ nanoparticles are in closest agreement with experimental XRD patterns.

The experimentally observed structural transition corresponds to the dashed pathway in Fig. 1. We attempted MD simulations of the experimental transition by adding water to the relaxed vacuum structure, but no transformation was observed. Within the time of the MD simulations, the structure was unable to find an accessible transition state.

\section{WAXS spectroscopy}


Experimental WAXS patterns were analyzed using specially written routines in IGOR-PRO, with a procedure following Refs 34, 35, 36 and 37, which is briefly reviewed here. We account for the detector deadtime $(\tau=2.77 \mu \mathrm{s})$ that causes the detected count rate, $I_{d}$, to lie below the true rate, $I$, as:

$$
I_{d}=\frac{1}{\tau}\left(1-e^{-I \tau}\right)
$$

After deadtime correction, the solvent or empty cell pattern is subtracted from the sample pattern. The background subtraction procedure is shown in Fig. 2. The experimental structure factor, $S(Q)$, is then obtained from:

$$
S(Q)=\frac{I(Q)}{\left\langle f^{2}(Q)\right\rangle}
$$

where $\left\langle f^{2}(Q)\right\rangle$ is the stoichiometric average of the atomic scattering factors, $f_{i}$, obtained from tabulated theoretical calculations [38]. A fit of $\left\langle f^{2}(Q)\right\rangle$ to the data in the region $10-35 \AA^{-1}$ provides data normalization (Fig. 2). We neglect the effects of Compton scattering and sample absorption, which are less than $5 \%$ for this experiment [34]. The reduced structure factor, $S(Q)-1$, is related to the real-space pair distribution function (PDF), or $G(r)$, by the Fourier sine transform:

$$
G(r)=\frac{2}{\pi} \int_{0}^{\infty} Q[S(Q)-1] \sin (Q r) d Q
$$

We include a Lanczos window function when performing this transform. For comparisons, theoretically calculated spectra (see below) were treated identically to experimental data.

Theoretical calculations of WAXS intensity were derived from the atomic coordinates of MD nanoparticle simulations. The same, MD frame-averaged atomic 
coordinates were used for WAXS and XAS calculations (below). WAXS spectra were calculated using the Debye equation [39]:

$$
I(Q)=\sum_{i} \sum_{j} f_{i} f_{j} \frac{\sin \left(Q r_{i j}\right)}{Q r_{i j}} \exp \left[-\frac{\sigma^{2} Q^{2}}{2}\right],
$$

where $\exp \left[-\sigma^{2} Q^{2} / 2\right]$ is the Debye-Waller (DW) thermal factor, and $\sigma^{2}$ is the atomic mean square displacement (MSD). Thermal motion reduces WAXS intensity, and a single average value of the MSD was obtained from a fit of the theoretical patterns to the appropriate data. Thermal damping of EXAFS is associated with the mean square relative displacement (MSRD) of atom pairs. MSD and MSRD are not identical because in MSRD the vibrations of an absorbing atom and its neighbors are correlated, particularly at low frequencies [40]. Hence, the MSRD is less than a sum of individual atom MSDs.

\section{EXAFS spectroscopy}

\section{i. Standard analysis}

The EXAFS signal, $\chi(k)$ was extracted using AUTOBK [42], and analyzed in FEFFIT [43] using $\mathrm{Zn}-\mathrm{X}(\mathrm{X}=\mathrm{S}, \mathrm{Zn}, \mathrm{O})$ scattering phase and amplitude functions calculated in FEFF 8.2 [26]. Individual scans were aligned before averaging using the first derivative of the reference $\mathrm{Zn}$ foil spectrum. The data were $k^{3}$-weighted for analysis in the range $k=2.5-11 \AA^{-1}$, and $R=1.2-2.8 \AA$ (single shell fits) or $R=1.5-4.5 \AA$ (three shell fits). The data were truncated because inclusion of higher- $k$ data introduced a low- $R$ shoulder in the first peak of the Fourier transform.

We extracted physical parameters from the EXAFS data that can be compared to the real-space MD predictions. The amplitude reduction factor, $S_{o}{ }^{2}$, was fit to data from 
the sphalerite reference and fixed for all subsequent analyses. Because it is impossible to determine the absolute energy of the excited photoelectron, the threshold position, $E_{o}$, is determined during EXAFS analysis by fitting. In order to achieve good agreement with the experimental spectra, $E_{0}$, was allowed to vary for every nanoparticle sample studied. This may reflect real electronic differences among the samples, but such observations are difficult to interpret quantitatively.

\section{ii. Asymmetric bond-length distributions}

In addition to standard EXAFS analysis, we performed fits of explicit asymmetric first-shell bond-length distributions. X-ray diffraction data and MD simulations indicate that the nanoparticles contain internal strain and have a wider bond length distribution than present in bulk ZnS. Asymmetric bond length distributions are commonly analyzed with the cumulant expansion of the EXAFS equation, but without uniquely defining a real-space distribution. To allow comparison with real-space structures derived from MD, we consider the generalized EXAFS equation:

$$
\chi(k)=\frac{N S_{o}^{2} F(k)}{k} \int_{0}^{\infty} \frac{g(r)}{r^{2}} e^{-2 \uparrow \lambda(k)} \sin (2 k r+\varphi(k)) d r
$$

where $N$ is the coordination number, $S_{o}{ }^{2}$ is the amplitude reduction factor, $F(k)$ is the photoelectron backscattering amplitude, $\varphi(k)$ is the phase shift due to the atomic potentials, $r$ is the bond length, and $k$ is the photoelectron wavevector. A convenient form of the pair distribution function, $g(r)$, is given by Yang et al. (Model II, Ref. 16)

$$
\begin{array}{ll}
g(r)=\frac{1}{D_{2}\left(r_{o}, s, \delta\right) \Gamma(s+1)} \frac{r^{2}}{\delta}\left(\frac{r-r_{o}}{\delta}\right)^{s} e^{-\left(\left(r-r_{o}\right)\right) / \delta}, & r \geq r_{o} \\
g(r)=0, & r<r_{o}
\end{array}
$$


where $r_{o}$ is the shortest bond length, and the parameters $s$ and $\delta$ define the shape of the distribution. $\Gamma(s+1)$ is the gamma function and $D_{2}$ can be found from the expression:

$$
D_{n}=\delta^{n}\left[\eta^{n}+\sum_{m=1}^{n}\left(\frac{n !}{m !(n-m) !}\right) \eta^{(n-m)} \prod_{j=1}^{m}(s+j)\right\rceil, \quad \eta=\frac{r_{o}}{\delta}
$$

This expression differs slightly from that published previously. A simple expression for the EXAFS equation is obtained upon integration:

$$
\chi(k)=\frac{N S_{o}^{2} F(k)}{k r_{o}^{2}} A_{c}(k) e^{-2 r_{o} / \lambda(k)} \sin \left(2 k r_{o}+\varphi(k)+\varphi_{c}\right) .
$$

Expressions for the amplitude and phase correction factors, $A_{c}(k)$ and $\varphi_{c}(k)$ are given in Ref. 16.

All stages of the first shell fits were performed using routines written in the analysis program IGOR-PRO, using the same $\mathrm{Zn}-\mathrm{S}$ scattering phase and amplitude functions as above for the standard analysis. To obtain the real-space first shell bond length distribution, the modified EXAFS expression (Eqn. 8) was fit to the backtransformed $k^{3}$-weighted data in the ranges $r=1.2-2.6 \AA$ and $k=2.5-11 \AA^{-1}$.

As a check of the analysis, the more usual EXAFS equation [15], including the first four cumulants, was fit to the data. We compared the best-fit values of the cumulants $\left(C_{1}\right.$ etc.) from the standard analysis to the cumulants obtained from the moments of the best-fit real-space distribution. For $g(r)$ given in (Eqn. 6), the relationships between the moments of the distribution and the cumulants are:

$$
\begin{aligned}
& C_{1}=\langle r\rangle=D_{3} / D_{2} ; \quad C_{2}=\left\langle r^{2}\right\rangle-\langle r\rangle^{2}=\left(D_{4} D_{2}-D_{3}^{2}\right) / D_{2}^{2} ; \\
& C_{3}=\left\langle r^{3}\right\rangle-3\left\langle r^{2}\right\rangle\langle r\rangle+2\langle r\rangle^{3}=\left(D_{5} D_{2}^{2}-3 D_{4} D_{3} D_{2}+2 D_{3}^{3}\right) / D_{2}^{3} ; \text { etc. }
\end{aligned}
$$


The cumulants derived from the standard and non-standard analyses agree to within $10-20 \%$. For this analysis, the amplitude reduction factor, $S_{o}^{2}$, and the energy threshold position, $E_{o}$, were fit to data from the sphalerite reference and were fixed for all subsequent analyses.

\section{iii. Whole-nanoparticle EXAFS calculations}

To determine whether the MD simulations reproduce the EXAFS data, we calculated whole-nanoparticle EXAFS spectra, using the same MD atomic coordinates as for WAXS and XANES calculations. In contrast to whole-particle XANES calculations described below, we made a single calculation of transferable scattering potentials, and used the CFAVERAGE card in FEFF [27].

The effects of thermal vibrations on EXAFS are dependent on the particular photoelectron scattering path. The correlated Debye model built into the FEFF code calculates the contribution on a path-by-path basis [47]. We tested a procedure in which thermal disorder parameters were obtained by fits to the EXAFS data and used as inputs for subsequent whole-nanoparticle EXAFS and XANES calculations. However, the correlated Debye model is not accurate for bulk $\mathrm{ZnS}$, even in the single scattering regime. We attempted to fit to bulk sphalerite and wurtzite data within $R=4.5 \AA$ of the EXAFS transform (approximately corresponding to $\mathrm{Zn}_{0}-\mathrm{S}_{1}, \mathrm{Zn}_{0}-\mathrm{Zn}_{2}$, and $\mathrm{Zn}_{0}-\mathrm{S}_{3}$ shells),

using three SS paths, varying a single Debye temperature, $\Theta_{\mathrm{d}}, S_{o}{ }^{2}$ and $E_{o}$, and bond lengths, at $300 \mathrm{~K}$, and fixing the coordination numbers at known values. The simulated EXAFS were a poor match to the data, over-estimating the contribution from $\mathrm{Zn}$ - Zn paths. The results were similar whether paths from sphalerite or wurtzite theoretical calculations were used. Inclusion of 3-leg multiple scattering paths had no effect on the fit. 
In contrast, a good fit to the data was obtained when the first three shells were given independent Debye temperatures (see Fig 8). To a good approximation, the bestfit Debye temperatures of the second and third shells are equal, and this is assumed in the calculations below. To obtain whole-particle EXAFS spectra, we performed three calculations. It was essential that each calculation averaged the EXAFS from every Zn atom in the nanoparticle because every site is unique. The desired EXAFS interference function, $\chi(k)$, was obtained from:

$$
\chi(k)=\chi\left\{Z n_{0} \rightarrow S_{3}^{M S} \mid \theta_{D}^{2}\right\}-\chi\left\{Z n_{0} \rightarrow S_{1}^{S S} \mid \theta_{D}^{2}\right\}+\chi\left\{Z n_{0} \rightarrow S_{1}^{S S} \mid \theta_{D}^{1}\right\}
$$

where, for example, $\chi\left\{Z n_{0}-S_{3}^{M S} \mid \theta_{D}^{2}\right\}$ indicates the total contribution for the first three shells (single and multiple scattering) calculated using the second shell Debye temperature $\theta_{D}^{2}$ (note that $\theta_{D}^{2}=\theta_{D}^{3}$ ).

In an alternative approach, MD simulations have been used to combine a statistically significant number of individual thermal configurations of periodic bulk materials [45] or hydrated ions [46]. However, this is impractical for a $3 \mathrm{~nm}$ nanoparticle containing $>700$ atoms.

\section{XANES spectroscopy}

We used frame-averaged atomic coordinates generated by MD simulations of $3 \mathrm{~nm} \mathrm{ZnS} \mathrm{nanoparticles} \mathrm{to} \mathrm{calculate} \mathrm{whole-nanoparticle} \mathrm{Zn} \mathrm{K} \mathrm{XANES} \mathrm{that} \mathrm{can} \mathrm{be}$ compared to the data. Provided x-ray absorption theory is adequate for this system, such calculations provide a new and stringent test of MD structure predictions. Unlike calculations of bulk crystalline materials, no atomic sites in nanoparticles are truly equivalent. We therefore calculated whole-nanoparticle $\mathrm{Zn}$ K XANES considering every $\mathrm{Zn}$ site independently (i.e. we calculated the scattering potentials for each site individually). We normalized the calculated XANES spectra and aligned them to a 
common Fermi energy before averaging. Because of the time consuming nature of this calculation, the only MD structure considered is that associated with water binding. We also calculated whole-nanoparticle Zn K XANES for the initial (unrelaxed) spherical $3 \mathrm{~nm}$ sphalerite crystallite by calculating the potentials for each concentric shell of $\mathrm{Zn}$ atoms. Previous work has shown that the calculation on Zn K XANES in ZnS converges within a cluster radius of $10 \AA$ [33], which we use as the cluster radius in the calculations performed here.

\section{i. Scattering potentials}

XANES calculations are very sensitive to the nature of the atomic scattering potentials. The self-consistent approach of FEFF 8 has been shown to be accurate for $\mathrm{ZnS}$. Published data and simulations for the compounds $\mathrm{ZnSO}_{4}$ and $\mathrm{ZnO}$ [31] indicate FEFF is also suitable for calculating the scattering potentials associated with $\mathrm{S}-\mathrm{O}$ and $\mathrm{Zn}-\mathrm{O}$ bonds. Adjustment of the interstitial potential as recommended in the FEFF manual [27] resulted in better agreement in peak positions between theoretical and experimental Zn K XANES of ZnS.

\section{ii. Exchange-correlation potential}

The exchange-correlation potential affects the energy spacing of XAS lineshape features, and incorporates the finite range of the photoelectron [48]. Previous work has shown the Hedin-Lundqvist local density exchange approximation to simulate all the major absorption edges of $\mathrm{ZnS}$ well [33]. In this work, we consider near-edge $\mathrm{Zn} \mathrm{K}$ absorption only, and, in agreement with Ref. 49, have found Dirac-Hara to be superior at this edge (the comparison is shown in Fig. 8).

To approximately include a thermal contribution for the calculation of XANES, we adjusted by hand $\theta_{D}$, and added uniform broadening, until the calculated XANES 
gave good agreement to data from the reference compounds. The best results of this procedure are shown in Fig. 8, and the calculation parameters are given in Table 2. A study of the thermal contributions to the XANES and EXAFS of ZnS will be the topic of a future manuscript.

\section{RESULTS}

\section{A. WAXS Analysis}

As shown in Fig. 3 (and Ref. 6), the addition of water to a suspension of uncoated $3 \mathrm{~nm} \mathrm{ZnS} \mathrm{nanoparticles} \mathrm{causes} \mathrm{a} \mathrm{profound} \mathrm{change} \mathrm{in} \mathrm{the} \mathrm{diffraction} \mathrm{pattern.} \mathrm{The}$ splitting and sharpening of diffraction peaks in the structure factor, $S(Q)$, show a reduction of structural disorder within the nanoparticles following water addition. The structure of hydrated nanoparticles more closely approaches that of bulk sphalerite, as seen from the appearance of the 220 and 311 diffraction peaks. From UV absorption measurements and TEM imaging, there is no coarsening of the nanoparticles following water addition [6]. Small angle x-ray scattering confirms this conclusion, and additionally indicates that the aggregation state is unchanged [50]. No x-ray scattering signal from water was observed in the real-space or reciprocal-space WAXS patterns [51].

Fig. 3 also shows that there is a very good agreement between experimental X-ray scatting data, including the real-space pair distribution function (PDF), and theoretical patterns based on the results of MD simulations. In view of the data, and the predictions of the MD simulations, we conclude that the structural transformation is a consequence of water binding to the nanoparticle surface. Experiments are presently ongoing to understand the nature of the water-nanoparticle interaction. 
For harmonic (thermal or static) disorder, a single, equilibrium real-space bond length generates a Gaussian peak shape in the PDF (implying a Gaussian distribution of bond lengths), plus finite-data termination ripples [52]. The Gaussian fits to the first shell of the WAXS PDF give $R_{\text {methanol }}=2.316 \AA, R_{\text {water }}=2.329 \AA$. The standard deviation of the Gaussian fits to PDF data is $\partial(R) \approx 0.001 \AA$. Thus, the relative difference in the $\mathrm{Zn}-\mathrm{S}$ bond lengths in methanol-coated nanoparticles and water-coated $\mathrm{ZnS}$ nanoparticles is $0.013 \AA$. However, it is probable that the real distribution of bond lengths in one or both materials is non-Gaussian. The presence of non-Gaussian bond length disorder is suggested by quality of fit for the methanol-coated nanoparticles, as measured by the statistic $\chi^{2}$, which is ten-times worse than for the water-coated nanoparticles. These observations suggest a real structural difference between the methanol- and water-coated particles. However, from the present PDF analysis, we cannot distinguish whether it arises due to difference in the average bond length or from differences in the details of the asymmetry of the bond length distribution.

\section{B. EXAFS analysis}

Experimental Zn K-edge EXAFS data and first shell fits for the nanoparticle and bulk samples are shown in Fig. 4. The fit results are summarized in Table 1. Nanoparticles that are in methanol or rapidly dried appear to have greater bond length disorder (MSRD) and asymmetry $\left(C_{3}\right)$ and appear to have lower first shell coordination than water-coated nanoparticles. Due to the correlation between MSRD and coordination number, the results in Table $\mathbf{1}$ are likely to reflect an overall increase in disorder and not a decrease in coordination.

The EXAFS fits included the possibility of non-Gaussian disorder and gave Zn-S bond lengths $R_{\text {methanol }}=2.349 \AA, R_{\text {water }}=2.348 \AA$. The bond length error estimated by the fitting code was $\partial(R) \approx 0.01 \AA$. These bond lengths differ from that obtained from 
those obtained from the PDFs by $0.020 \AA$ for $R_{\text {methanol }}$ and $0.026 \AA$ for $R_{\text {water }}$. This discrepancy may be due to the neglect of non-Gaussian disorder in the PDF analysis or to systematic errors [15]. For the reference sphalerite sample, the EXAFS fits give $R_{\text {sphalerite }}=2.347 \AA$. Thus, EXAFS data do not confirm the bond length contraction for methanol-coated nanoparticles, and there is no evidence for bond length contraction in the nanoparticles relative to bulk material.

Studies on tetrahedrally-coordinated semiconductor nanoparticles have found that the first shell bond length may be modified $[9,10,11]$ or unchanged $[12,13,14]$ with respect to the bulk material. Rockenburger et al. concluded that the nature of the surface ligand determined the direction of bond length changes [9]. Covalently attached ligands caused an expansion, while ligands that were non-specifically bound via ionic ligands led to bond length contraction. Bond length contraction is often taken to indicate the presence of an internal excess (Gibbs) pressure, resulting from surface relaxation and reconstruction. However, isotropic strain models are inadequate. A many-shell PDF analysis of nanocrystalline diamond showed no simple relationship between the distance shifts of the first 8 shells (most shells showed contraction, but one showed expansion) [18]. Similarly, Carter et al. report a first shell contraction and a second shell expansion from EXAFS analysis of CdSe [11]. In general, small particle size and the presence of bond-bending disorder prevents meaningful structure analysis of second and higher shells in EXAFS data from nanoparticles.

First shell analysis alone is thus insufficient to describe the structural modifications found in small particles. However, it is a clear test of the predictions of MD simulations. If all testable aspects of the MD predictions agree with experimental data, additional structural details may be taken with more confidence from the simulations. As described in the Methods, we fitted explicit real-space bond-length distributions to the bulk and nanoparticle EXAFS data. Fig. 5 shows the comparison 
between the fitted bond-length distributions and the MD predictions of the nanoparticle $\mathrm{Zn}-\mathrm{S}$ bond length in vacuum and with surface water. The MD simulations predict a bond length contraction for the nanoparticle in vacuum. By contrast, the experimental real-space data from EXAFS do not show any significant contraction in the structure of nanoparticles in methanol relative to nanoparticles in water. This conclusion is not affected by the neglect of thermal motion in the generation of the MD bond-length distribution.

We have assumed that simulated structures of nanoparticles in vacuum can be compared to experimentally observed structures from nanoparticles in methanol. The discrepancy between the experimental and predicted bond-length distributions (Fig. 5) may indicate that this assumption is not valid. We attempted to determine whether neglect of methanol in the simulations would lead to a significant change in the structure prediction. It was not possible to do this by obtaining the PDF from isolated nanoparticles in vacuum. Consequently, we used two alternative datasets to investigate the effect of methanol on $\mathrm{Zn}-\mathrm{S}$ bond lengths.

First, we examined $\mathrm{ZnS}$ nanoparticles that were rapidly vacuum dried and measured in dry nitrogen at room temperature. These particles are anticipated to have surfaces coated with residual methanol and/or nitrogen. The WAXS pattern from this sample is very similar to the pattern from the suspension in methanol (Fig. 6), indicating no significant change in the $\mathrm{Zn}-\mathrm{S}$ bond length associated with methanol removal.

Second, we acquired S K edge EXAFS spectra on ZnS nanoparticles synthesized in methanol, after room temperature vacuum drying, and after methanol desorption at 50 ${ }^{\circ} \mathrm{C}$ and $2 \times 10^{-6}$ Torr. As shown in Fig. 7, there is a significant change in the EXAFS transform associated with methanol desorption (this is a reversible effect, [6]). Thus, structural effects clearly do occur when methanol is removed. This implies that 
methanol-ZnS interactions are significant in stabilizing nanoparticle structure. Although we cannot fully explain the relative contributions of disorder, coordination number changes, and asymmetry to the spectrum in Fig. 7, there was no evidence of first-shell bond-length contraction. In summary, the absence of bond length changes upon methanol removal support our decision to neglect surface methanol in MD simulations as a first approximation.

\section{Whole-nanoparticle XAS calculations}

Further evaluation of the structures predicted by MD are made by performing whole-nanoparticle calculations of XAS spectra. To compare experimental and theoretical XAS spectra it is important to consider thermal broadening. This is accomplished semi-empirically (see Methods). The results of the EXAFS and XANES calculations and the experimental data for the bulk reference materials are given in Fig.

8.

\section{Pure sphalerite structure model nanoparticle}

Fig. 9 shows the whole-nanoparticle calculations of Zn K-edge XANES and EXAFS (the transform is shown). In the EXAFS transform, the calculated spectrum of the initial (unrelaxed) $3 \mathrm{~nm}$ nanoparticle is indistinguishable from the calculated bulk sphalerite spectrum. By contrast, the XANES spectrum of the initial (unrelaxed) nanoparticle shows significant broadening with respect to the bulk. The model calculations for the unrelaxed particle therefore indicate that EXAFS is not sensitive to particle size (for $3 \mathrm{~nm}$ particles) in the absence of disorder, while XANES is apparently sensitive to particle size. As discussed below, both EXAFS and XANES are sensitive to disorder. 
The calculated EXAFS transform for the unrelaxed nanoparticle gives relatively poor agreement to the experimental data for the water-coated nanoparticle, particularly in over-estimating the second shell contribution. This is apparent when the curve predicted for $3 \mathrm{~nm}$ undistorted sphalerite is compared to the experimental curve shown in the inset to Fig. 9. This indicates that there is disorder in the nanoparticles, particularly bond-bending disorder [13], which reduces the second shell contribution to the EXAFS transform. In apparent contradiction, the same unrelaxed nanoparticle gives relatively good agreement with the experimental water-coated nanoparticle data in the XANES spectrum.

\section{MD simulations of nanoparticle structure}

We calculated the EXAFS transforms for the MD-predicted water-coated and uncoated nanoparticle structures. The MD simulations reproduce the experimental trend toward higher crystallinity with water adsorption, as indicated by enhancement of the second shell contribution following water-binding (arrow, Fig. 9). However, the magnitude of the calculated EXAFS signal for both water-coated and uncoated nanoparticles is significantly lower than that observed in the experimental data (inset to Fig. 9). It is notable that the calculated XANES for the water-coated nanoparticle shows extreme lineshape broadening and is in very poor agreement with experimental data.

\section{DISCUSSION}

MD energy minimization accurately predicts the general effect of water binding on the structure of $3 \mathrm{~nm} \mathrm{ZnS} \mathrm{nanoparticles,} \mathrm{and} \mathrm{gives} \mathrm{very} \mathrm{good} \mathrm{quantitative} \mathrm{agreement}$ with WAXS data. However, EXAFS and XANES calculations for the same MD structures show much less agreement with the corresponding experimental data. In particular, the EXAFS and XANES calculations indicate the presence of excess 
structural disorder in the MD simulations. Therefore, either the XAS measurements are sensitive to structural characteristics absent from the MD structures, or the theory used for whole-nanoparticle EXAFS and XANES simulation is inadequate.

In the case of XANES, which gave poorest agreement, an important ingredient is missing from the theory. The neglect of a potential step (work function) at the surface of the nanoparticles means that low energy photoelectrons are not confined within the nanoparticle close to the absorption threshold. Surface potential barriers have been included in energy electron diffraction theory [53]. Long range multiple scattering is well known to be very important in determining accurate lineshape in the near edge region [48]. Inclusion of a surface barrier that confines the low energy scattered photoelectrons would reinforce scattering and interference close to absorption edge. If this was taken into consideration, the XANES of a model undistorted nanoparticle would show less lineshape broadening, thus much less dependence on size (Fig. 9).

In contrast to XANES calculations, the whole nanoparticle EXAFS calculations only require consideration of the three nearest neighbor shells, and consequently are much less sensitive to the confining surface. The EXAFS calculations based on the MD simulations did reproduce some important features of the experimental data. For example, in both theory and experiment, only the first shell contribution was observed in the room temperature EXAFS transform for nanoparticles without water binding. This results from extensive cancellation of scattered photoelectron contributions due to strong disorder [54]. However, some important discrepancies are clear. The simulated EXAFS amplitudes were considerably lower than observed, indicating that the MD simulations predict too much disorder.

Fig. 7 shows that methanol desorption may increase internal distortion. The associated reduction in the first shell S-Zn peak height brings the experimental data 
closer to the peak heights predicted for nanoparticles in vacuum. However, even in the presence of water, EXAFS calculations indicate that the MD structures are too distorted (Fig. 9). This could be a consequence of the limited number of water molecules used in the simulation ( 86 water molecules for $\sim 190 \mathrm{Zn}$ and $\sim 190 \mathrm{~S}$ surface atoms). Increasing the water coverage may improve the agreement with the experimental data.

It is surprising that a structure obtained from MD simulations can give good agreement with experimental WAXS data, yet poor agreement with experimental EXAFS data. This implies that certain details of the structure have only a weak influence on WAXS but strongly affect the EXAFS signal. As described below, we infer that, in contrast to WAXS, EXAFS is sensitive to the distribution of static disorder.

To demonstrate this point, a spherical $2.5 \mathrm{~nm}$ nanoparticle cut from a perfect sphalerite lattice was subjected to random atomic displacements, with the magnitudes of the displacements satisfying a Gaussian atomic position probability distribution function (consistent with harmonic vibrations [40]). In three runs, all atoms could be moved with equal probability; in three further runs, atoms farther from the center underwent larger displacements. The final total mean squared displacements (MSD), summed over the whole particle, and relative to the initial structure, are the same in every run $\left(\sum \underline{u}_{i}^{2} \approx 0.032 \AA^{2}\right)$, and the displacements themselves sum to zero $\left(\sum \underline{u}_{i} \approx 0\right)$. The MSD was chosen to give $\mathrm{Zn}-\mathrm{S}$ bond length distributions similar in width to the MD nanoparticle simulation in vacuum. The resulting theoretical WAXS and EXAFS spectra are given in Figs. 10 a \& b. In contrast to previous calculations of EXAFS, only first shell contributions are considered. The $Q$-weighted WAXS structure factors for all runs are indistinguishable, while clear differences between all $k^{3}$-weighted EXAFS spectra are seen. Furthermore, the amplitudes of the EXAFS spectra are systematically 
lower for model nanoparticles subject to uniform compared to surface-weighted atomic displacements.

Applying the observation that more non-uniform disorder gives stronger EXAFS intensity (and vice versa), the combined WAXS and EXAFS results suggest that the disorder within the model nanoparticles should be more partitioned than predicted in the MD. That is, better agreement with the nanoparticle EXAFS data would be obtained if there were greater disorder (or reconstruction) at the surface and more periodicity in the interior. This would lead to better agreement in the real-space RDFs obtained from bond length fitting (Fig. 5) and in the theoretical and experimental whole-nanoparticle EXAFS transforms (Fig. 9). An atomic rearrangement that achieved this would maintain good agreement with the WAXS data if the overall structural disorder (relative to perfect sphalerite) were unchanged. This conclusion is in agreement with other groups who have proposed that surface reconstruction leads to a core-shell geometry within nanoparticles [18].

WAXS and EXAFS interference functions are similar in origin, and both may be expressed as a sum of interference terms over atom pairs. For WAXS every pair contributes, while for each absorber atom in EXAFS only short range near-neighbors contribute due to the finite mean free path of the photoelectron. The near-neighbor contribution can be isolated from WAXS data by back-transforming the first-shell peak in the real-space PDF. As expected, when plotted with $Q^{3}$-weighting, Fig 10 c shows that this partial structure factor shows a similar sensitivity to the distribution of disorder and to the particular set of atom coordinates as the first shell EXAFS spectrum (Fig 10 b). Data quality precludes such a treatment of the experimental WAXS data in this case.

\section{CONCLUSIONS}


MD simulations are used to investigate nanoparticle structure, producing specific predictions about interior and surface structure that would be a natural starting point for subsequent calculations of electronic properties and reactivity. However, validation of MD structures is essential before such predictions can be used with confidence. As theoretical methods to simulate $\mathrm{x}$-ray absorption spectroscopies are now readily available, it is attractive to use them as complementary structural analyses to x-ray diffraction. Combined whole-particle EXAFS and WAXS studies are a more stringent test of nanoparticle structure predictions than WAXS alone, although we conclude that further theory development is required for multiple scattering calculations of XANES for nanoscale systems. XANES calculations suffer excessive broadening of fine structure due to the absence of a confining surface potential barrier, but this does not affect EXAFS calculations. Furthermore, as a sum of local measurements, EXAFS is more sensitive than WAXS to the distribution of static disorder (unless WAXS data is treated unconventionally). As a consequence, EXAFS data could provide additional constraints for structure analysis.

Molecular dynamics simulations predict that the binding of water to $\mathrm{ZnS}$ nanoparticle surface produces a significant increase in crystallinity. Experimental WAXS, EXAFS and XANES firmly corroborate this trend. By combining EXAFS and WAXS data and MD simulations, we conclude that structural disorder is strongly partitioned between interior and surface regions.

Acknowledgments: Thanks to Grant Bunker, Mark Beno, and Jacob Urquidi. We thank Paul Alivisatos for access to equipment and Dr. W. Smith, Dr. T.R. Forester and Dr. D. Fincham for providing MD codes. S K edge EXAFS data were acquired on the DCM beamline of the Canadian Synchrotron Radiation Facility (CSRF) at the UW-Madison Synchrotron Radiation Center (SRC). The CSRF is supported by the Natural Sciences and Engineering Council (NSERC) of Canada and the National Research Center Canada (NRC-CRNC) and the SRC is supported by Division of Materials Research of 
the US National Science Foundation, under Award No. DMR-0084402. WAXS data were acquired on beamline 11-ID-C, and Zn K edge EXAFS data on beamline 4-ID-D, at the Advanced Photon Source (APS). Use of the Advanced Photon Source was supported by the U. S. Department of Energy, Office of Science, Office of Basic Energy Sciences, under Contract No.W-31-109-Eng-38. HRTEM was performed at the National Center for Electron Microscopy, Berkeley, CA, and we thank Chengyu Song. Financial support for this work came from the US Department of Energy (DOE), a Lawrence Berkeley National Lab. LDRD, and the US National Science Foundation (NSF).

\section{References}

1. A. P. Alivisatos, Science, 271, 933 (1996).

2. C. B. Murray, D. J. Norris, and M. G. Bawendi, J. Am. Chem. Soc. 115, 8706 (1993).

3. J. M. McHale, A. Auroux, A. J. Perotta and A. Navrotsky, Science 277, 788 (1997).

4. H. Zhang and J. F. Banfield, J. Mater. Chem. 8, 2073 (1998).

5. G. J. Ackland. Rep. Prog. Phys. 64483 (2001).

6. H. Zhang, B. Gilbert, F. Huang and J. F. Banfield, Nature 424, 1025 (2003).

7. F. Huang, B. Gilbert, H. Zhang and J. F. Banfield. Phys. Rev. Lett. in press.

8. S. B. Qadri, E. F. Skelton, D. Hsu, A. D. Dinsmore, J. Yang, H. F. Gray, and B. R. Ratna, Phys. Rev. B 60, 9191 (1999).

9. J. Rockenberger, L. Tröger, A. L. Rogach, M. Tischer, M. Grundmann, A. Eychmüller and H. Weller, J. Chem. Phys. 108, 7807 (1998).

10. J. Rockenberger, L. Tröger, A. Kornowski, T. Vossmeyer, A. Eychmüller, J. Feldhaus and H. Weller, J. Phys. Chem. B 101, 2691 (1997). 
11. A. C. Carter, C. E. Bouldin, K. M. Kemner, M. I. Bell, J. C. Woicik and S. A. Majetich, Phys. Rev. B 55, 13822 (1997).

12. M. A. Marcus, W. Flood, M. Steigerwald, L. Brus and M. Bawendi. Structure of capped CdSe cluster by EXAFS. J. Phys. Chem. 95, 1572 (1991).

13. M. A. Marcus, L. E. Brus, C. Murray, M. G. Bawendi, A. Prasad and A. P. Alivisatos. Nanostructured Materials 1, 323 (1992).

14. H. Hosokawa, H. Fujiwara, K. Murakoshi, Y. Wada, S. Yanagida and M. Satoh. J. Phys. Chem. 100, 6649 (1996).

15. E. D. Crozier, J. J. Rehr \& R. Ingalls, in X-ray Absorption: Principles, Applications, Techniques of EXAFS, SEXAFS and XANES, edited by D. C. Konigsberger and R. Prins. Wiley, New York, 1988.

16. D. S. Yang, D. R. Fazzini, T. I. Morrison, L. Tröger and G. Bunker. J. NonCrystalline Solids 210, 275 (1997).

17. F. Dassenoy, M.-J. Casanove, P. Lecante, M. Verelst, E. Snoeck, A. Mosset, T. Ould Ely, C. Amiens and B. Chaudret. J. Chem. Phys. 112, 8137 (2000).

18. B. Palosz, I, E. Grzanka, S. Gierlotka, S. Stel'makh, R. Pielaszek, U. Bismayer, J. Neuefeind, H.-P. Weber, Th. Proffen, R. Von Dreele and W. Palosz, Z. Kristallogr. 217, 497 (2002).

19. K. Leung and K. B. Whaley, J. Chem. Phys. 110, 11012 (1999).

20. E. Rabani, J. Chem. Phys. 115, 1493 (2001).

21. A. Puzder, A. J. Williamson, F. A. Reboredo, and G. Galli, Phys Rev Lett. 91 157405 (2003).

22. K. Wright and R. A. Jackson, J. Matter. Chem. 5, 2037 (1995). 
23. K. Wright, G. W. Watson, S. C. Parker and D. J. Vaughan, Am. Mineral. 83, 141 (1998).

24. S. Hamad, S. Cristol, and C. R. A. Catlow, J. Phys. Chem. B 106, 11002 (2002).

25. H. Zhang, F. Huang, B. Gilbert and J. F. Banfield, J. Phys. Chem. B 107, 13051 (2003).

26. A. L. Ankudinov, B. Ravel, J. J. Rehr and S. D. Conradson. Phys. Rev. B 587565 (1998).

27. A. L. Ankudinov, B. Ravel, and J. J. Rehr. FEFF 8.0 Manual, 2002. Online at: http://leonardo.phys.washington.edu/feff/

28. D. J. Harris, J. P. Brodholt, J. H. Harding and D. M. Sherman. Molecular Physics 99, $825(2001)$

29. J. E. Stevens, R. K. Chaudhuri and K. F. Freed. J. Chem. Phys. 105, 8754 (1996).

30. N. H. de Leeuw and S. C. Parker. Phys. Rev. B 58, 13901 (1998).

31. G. A. Waychunas, C. C. Fuller, J. A. Davis and J. J. Rehr. Geochim. Cosmochim. Acta 67, 1031 (2003).

32. A. L. Ankudinov, J. J. Rehr, J. J. Low and S. R. Bare. J. Chem. Phys. 116, 1911 (2002).

33. B. Gilbert, B. H. Frazer, H. Zhang, F. Huang, J. F. Banfield, D. Haskel, J. C. Lang, G. Srajer and G. De Stasio. Phys. Rev. B 66, 245205 (2002).

34. J. Urquidi, C. J. Benmore, J. Neuefeind and B. Tomberli. J. Appl. Cryst. 36, 368 (2003).

35. B. J. Thijsse. J. Appl. Cryst. 17, 61 (1984). 
36. I.-K. Jeong, J. Thompson, Th. Proffen, A. Perez and S. J. L. Billinge. PDFgetX, a program for obtaining the pair distribution function from x-ray powder diffraction data.

37. A.C. Wright in Amorphous insulators and semiconductors. Edited by M.F. Thorpe and M.I Mitkova. pp 83-131. Dordrecht: Kluwer.

38. J. H. Hubbell, Wm. J. Viegele, E. A. Briggs, R. T. Brown, D. T. Cromer \& R. J. Howerton. J. Phys. Chem. Ref. Data 4, 471, 1975.

39. Guinier, A. X-ray diffraction in crystals, imperfect crystals and amorphous bodies. W.H. Freeman \& Co., San Francisco, 1963.

40. B.T.M. Willis \& A.W. Pryor. Thermal vibrations in crystallography. Cambridge University Press, Cambridge, 1975.

41. I.-K. Jeong, Th. Proffen, F. Mohiuddin-Jacobs and S. J. L. Billinge. J. Phys. Chem. A 103921 (1999).

42. B. Ravel, J. Synchrotron Rad. 8, 314 (2001).

43. E. A. Stern, M. Newville, B. Ravel, Y. Yacoby and D. Haskel. Physica B 117, 208 (1995).

44. R. E. Benfield, D. Grandjean, M. Kröll, R. Pugin, T. Sawitowski \& G. Schmid. J. Phys. Chem. B 105, 1961 (2001).

45. A. Filipponi, F. Evangelisti, M. Benfatto, S. Mobilio and C. R. Natoli, Phys. Rev. B 409636 (1989).

46. F. Jalilehvand, D. Spångberg, P. Lindqvist-Reis, K. Hermansson, I. Persson and M. Sandström. J. Am. Chem. Soc. 123, 431 (2001)

47. E. Sevillano, H. Meuth and J. J. Rehr. Phys. Rev. B. 20, 4908 (1979).

48. J. J. Rehr and R. C. Albers. Rev. Mod. Phys. 72, 621 (2000). 
49. Ph. Sainctavit, J. Petiau, M. Benfatto, and C. R. Natoli, Physica B 158, 3470 (1989).

50. B.Gilbert, H. Zhang, F. Huang and J. F. Banfield, unpublished data.

51. T. Head-Gordon and G. Hura. Chem. Rev. 102, 2651 (2002).

52. J.S. Chung and M.F. Thorpe. Phys. Rev. B 55, 1545 (1997).

53. M. A. van Hove, W. H. Weinberg, C.-M. Chan (Eds). Low-Energy Electron

Diffraction: Experiment, Theory and Surface Structure Determination, Springer (1986).

54. P. A. O’Day, J. J. Rehr, S. I. Zabinsky and G. E. Brown, Jr. J. Am. Chem. Soc. 116, 2938 (1994). 


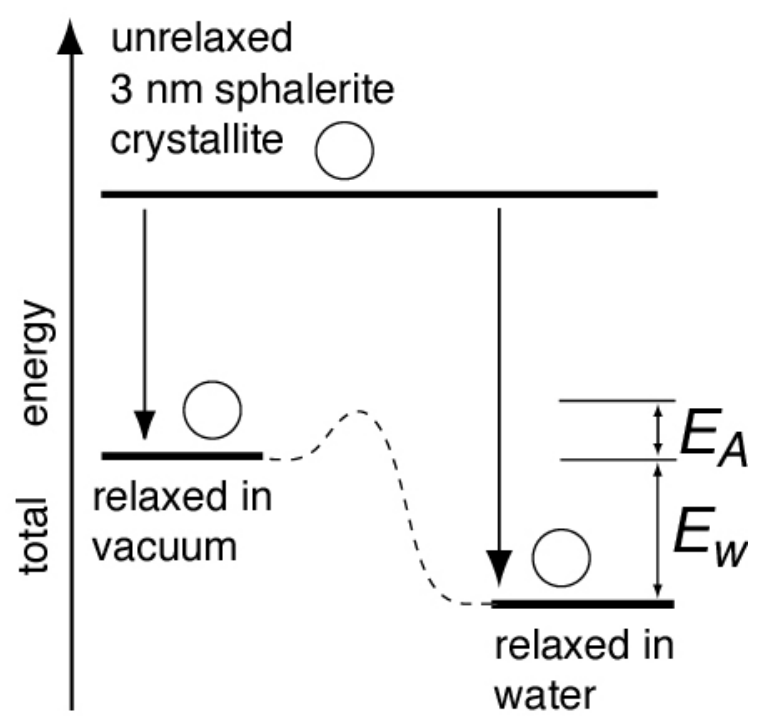

Figure 1. 


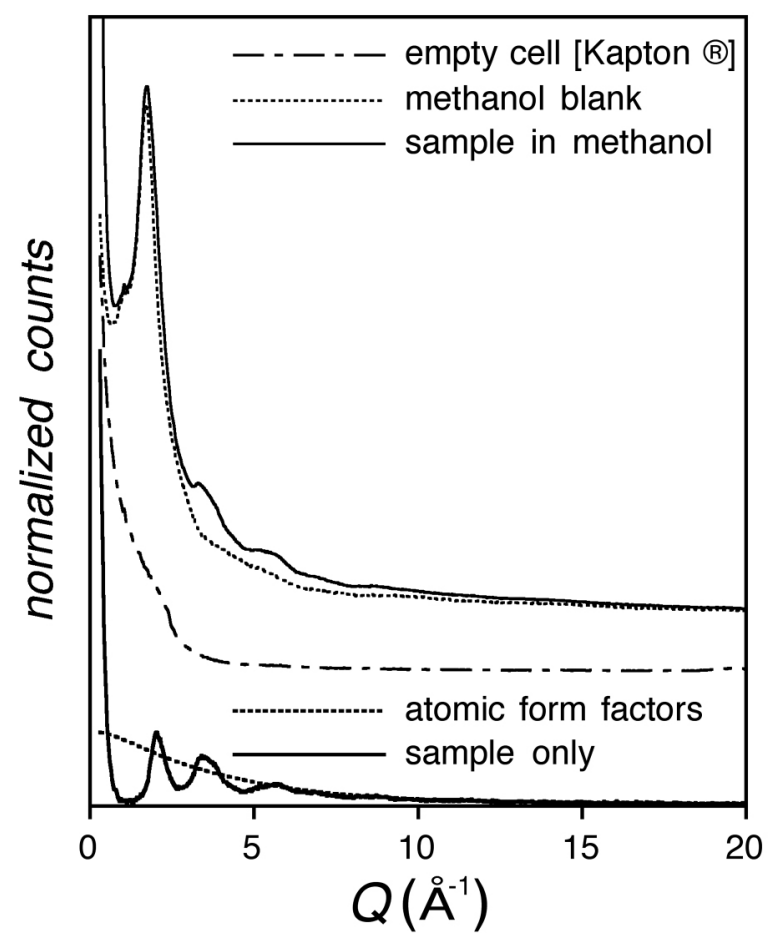

Figure 2. 

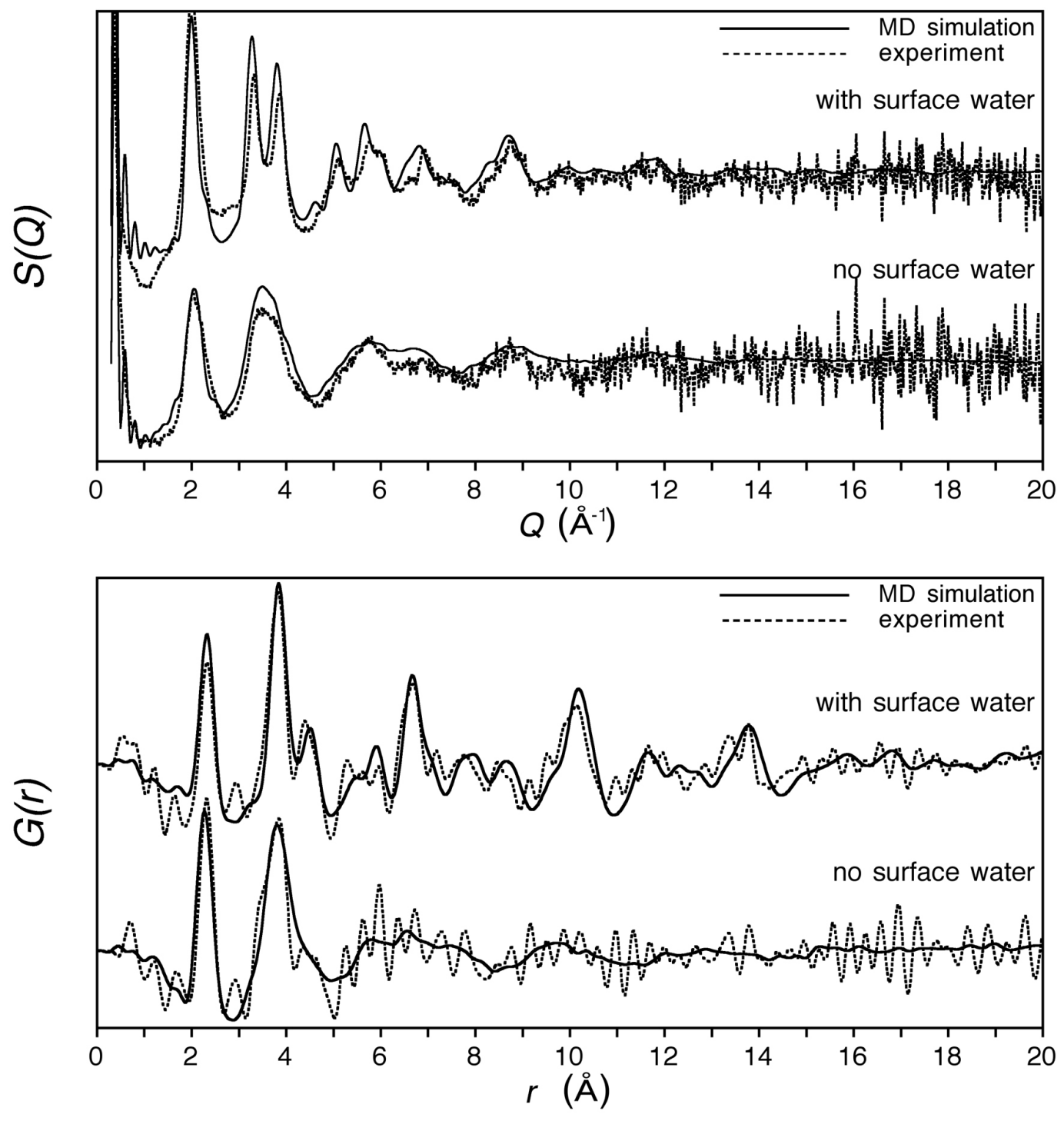

Figure 3. 

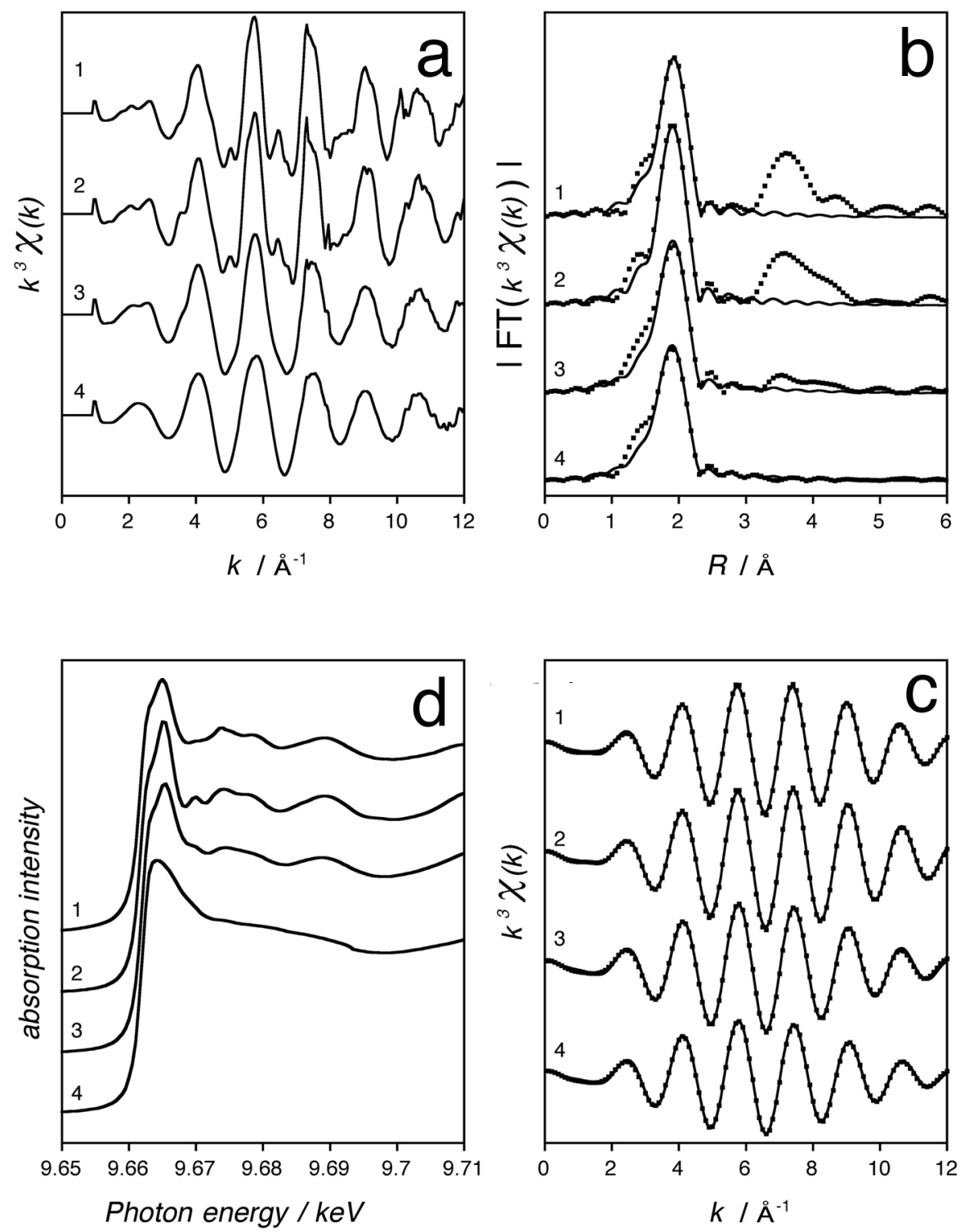

Figure 4. 


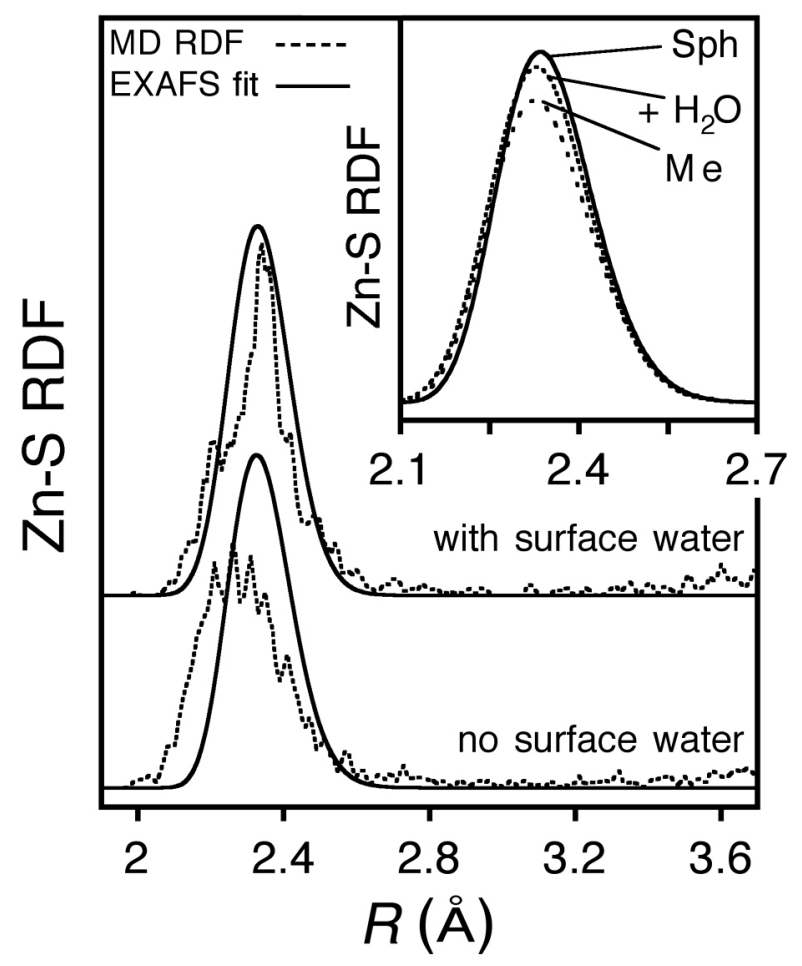

Figure 5. 

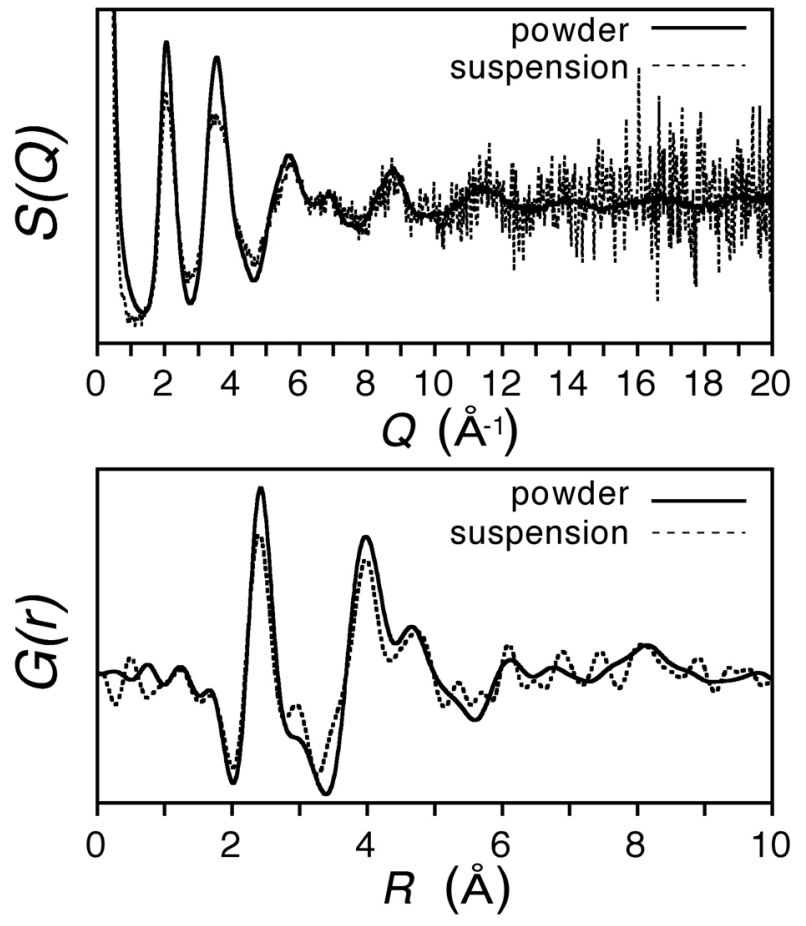

Figure 6. 


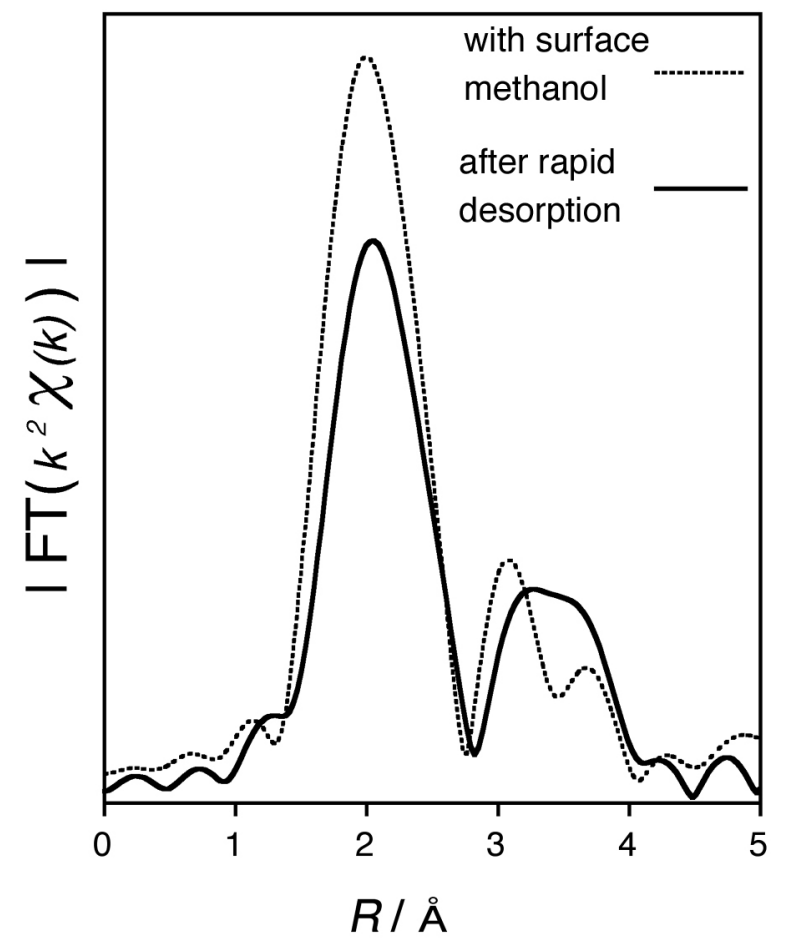

Figure 7. 

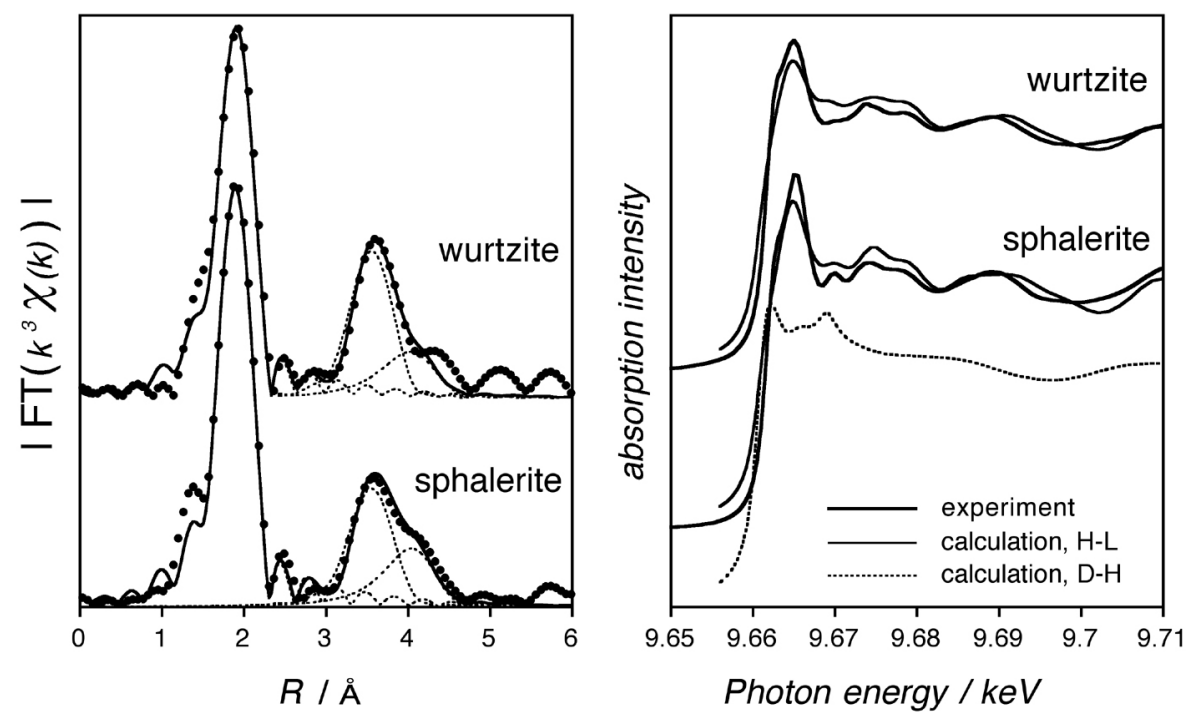

Figure 8. 

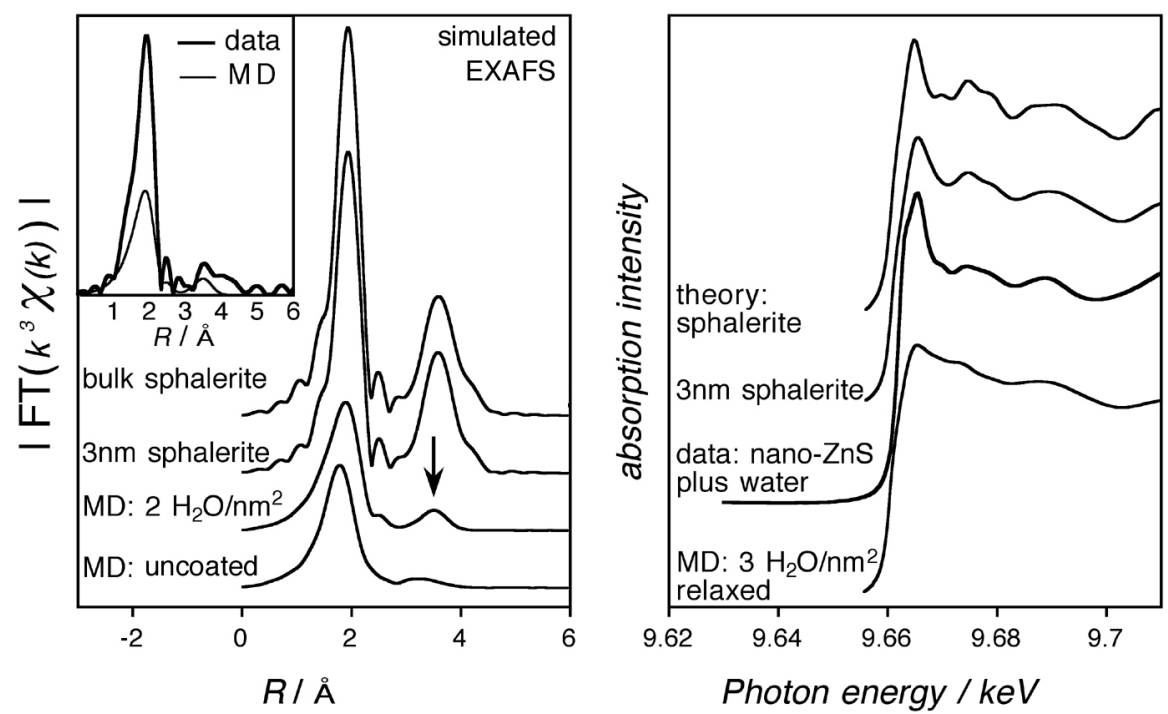

Figure 9. 

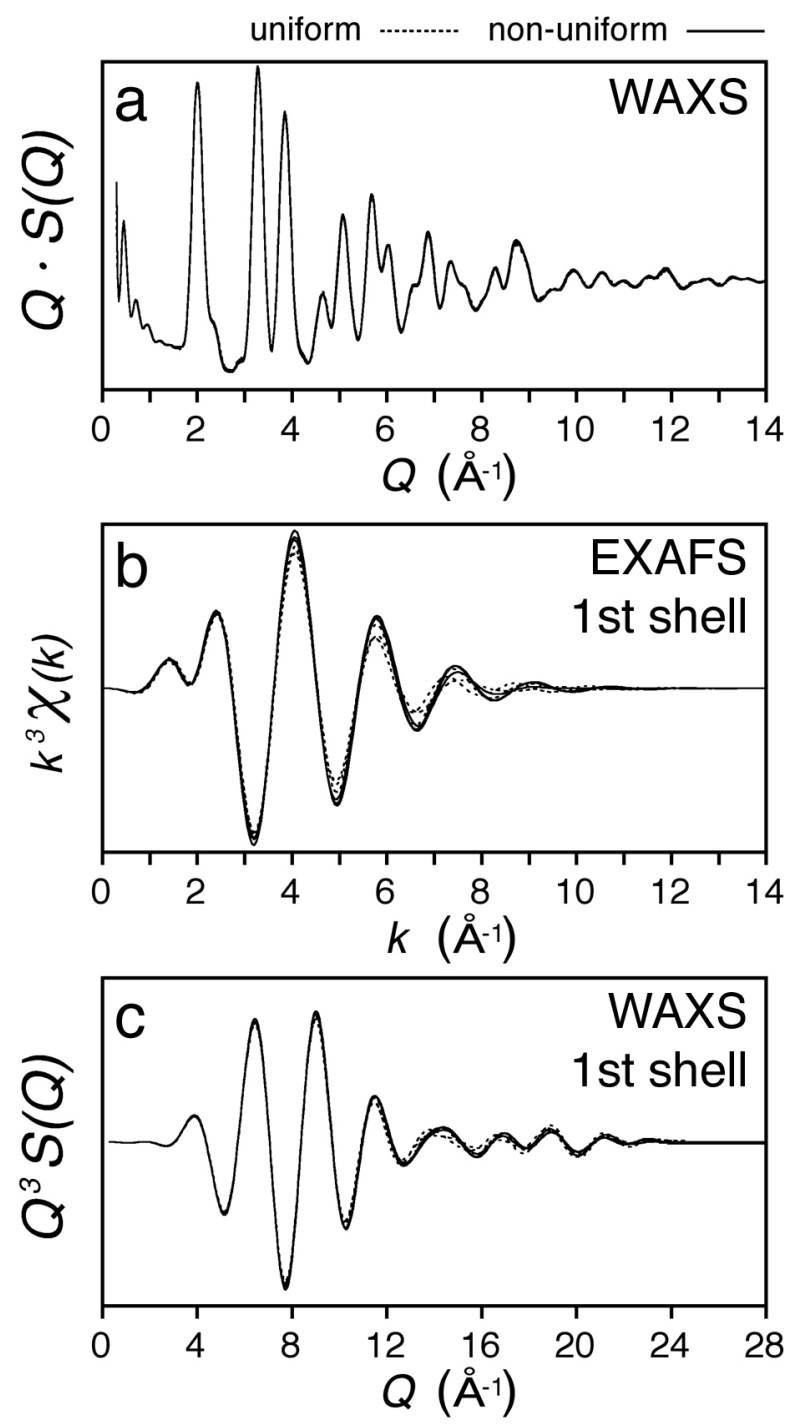

Figure 10. 


\begin{tabular}{l|l|l|l|l} 
& sphalerite & $\begin{array}{l}\text { nano } \mathrm{ZnS} \\
\text { in } \mathrm{MeOH}\end{array}$ & $\begin{array}{l}\text { nano } \mathrm{ZnS} \\
+\mathrm{H}_{2} \mathrm{O}\end{array}$ & $\begin{array}{l}\text { nano } \mathrm{ZnS} \\
\mathrm{DRY}\end{array}$ \\
\hline$\Delta E(\mathrm{eV})$ & 0.33 & 0.0 & 0.26 & 0.51 \\
$\mathrm{CN}$ & {$[4]$} & 3.6 & 4.0 & 3.3 \\
$R(\AA)$ & 2.347 & 2.349 & 2.348 & 2.354 \\
$\mathrm{MSRD}^{2}\left(10^{-3} A^{2}\right)$ & 5.1 & 7.0 & 6.8 & 7.8 \\
$C_{3}\left(10^{-4} \AA^{2}\right)$ & {$[0]$} & 3.0 & 2.3 & 2.7 \\
$\chi^{2}$ & 944 & 10900 & 3181 & 848 \\
\hline
\end{tabular}

Table 1. 


\begin{tabular}{|c|c|c|c|}
\hline \multicolumn{4}{|c|}{ EXAFS Parameters } \\
\hline & $\Theta_{\mathrm{D} 1} / \mathrm{K}$ & $\Theta_{\mathrm{D} 2} / \mathrm{K}$ & $\Theta_{\mathrm{D} 3} / \mathrm{K}$ \\
\hline Sphalerite & 433.1 & 262.0 & 264.2 \\
\hline Wurtzite & 476.0 & 246.5 & 285.2 \\
\hline \multicolumn{4}{|c|}{ XANES Parameters } \\
\hline & & $\Theta_{D} / K$ & 200 \\
\hline $\mathrm{R}_{\mathrm{scf}} / \AA$ & 4 & $\mathrm{R}_{\mathrm{fms}} / \AA$ & 10 \\
\hline $\mathrm{I}_{\mathrm{scf}} / \AA$ & 2 & $\mathrm{I}_{\mathrm{fms}} / \AA$ & 2 \\
\hline$\Delta \mathrm{E}_{\mathrm{F}} / \mathrm{eV}$ & 3.0 & $\Delta \mathrm{E}_{\mathrm{i}} / \mathrm{eV}$ & 0.0 \\
\hline
\end{tabular}

Table 2. 
Figure 1. Energetic state diagram for the molecular dynamics simulations performed in this work. Spherical model nanoparticles cut from a sphalerite lattice were dynamically relaxed with or without surface water. The experimental transition pathway is shown by the dashed line. $E_{A}$ is the (unknown) activation energy; $E_{W}$ is enthalpy of water binding and lattice rearrangement. From MD simulations, $E_{w} \sim 500 \mathrm{~kJ} / \mathrm{mol} \mathrm{H}_{2} \mathrm{O}$ at $3 \mathrm{H}_{2} \mathrm{O} / \mathrm{nm}^{2} \mathrm{ZnS}$ surface coverage [6].

Figure 2. Solvent and window background subtraction from in situ wide angle Xray scattering data from a suspension of $\mathrm{ZnS}$ nanoparticles in methanol. All spectra at the same scale, but resulting sample data and fitted atomic form factors have been displaced for clarity.

Figure 3. Wide angle X-ray scattering observation of the water-driven structural transition. Q-range $=0.3-22 \AA^{-1}$. Experimental data (dotted lines) are compared with theoretical curves derived from MD simulation (solid lines). Top: Structure factor, $S(Q)$; Bottom: pair distribution function, $G(r)$. Curves are displaced for clarity.

Figure 4. Experimental Zn K-edge X-ray absorption spectra from bulk and nanocrystalline ZnS. a) $k^{3}$-weighted EXAFS spectra; b) magnitude of the Fourier transform, including fit to first shell (data = symbols, fit = line); c) backtransformation of first shell and fit $($ data $=$ symbols, fit $=$ line $)$. $d)$ XANES spectra. Key to spectra: 1 . Wurtzite reference. 2 . Sphalerite reference. 3 . Nanocrystalline $\mathrm{ZnS}$ in methanol following addition of water. 4. Nanocrystalline ZnS in methanol without additional water. 
Figure 5. Nanoparticle Zn-S partial radial distribution functions (Zn-S RDF) with and without surface water from fits to Zn K-edge EXAFS data, compared with RDFs from structures predicted by MD simulation. The curves have been displaced for clarity. The thermal motion of MD atoms was not considered. Inset: The nanoparticle RDFs and the bulk sphalerite (Sph) RDF superimposed at the same scale.

Figure 6. Wide angle X-ray scattering data from $3 \mathrm{~nm} Z \mathrm{nS}$ nanoparticles suspended in methanol and the powder obtained from rapidly drying the suspension at room temperature. The data from the dry powder is higher quality than from the suspension. However, there is no detectable structural consequence of drying. The dry powder data were acquired and treated identically to the data in Fig. 1.

Figure 7. S K-edge EXAFS transforms from powders of $3 \mathrm{~nm} Z \mathrm{nS}$ nanoparticles in vacuum, before and after thermal desorption of methanol. The reduction in the first shell $(S-Z n)$ peak height indicates that methanol desorption increases structural disorder.

Figure 8. Theoretical calculations of room temperature Zn K EXAFS (left) and XANES (right) from bulk wurtzite and sphalerite reference compounds, including the effects of thermal disorder. The EXAFS calculations include only the three nearest neighbor shells. The best fit EXAFS Debye Temperatures, and the parameters for the XANES calculations are given in Table 2. The HedinLundqvist $(\mathrm{H}-\mathrm{L})$ exchange potential gives better results than the Dirac-Hara (D- 
H) potential for Zn K XANES calculations for bulk sphalerite and wurtzite (comparison for wurtzite not shown).

Figure 9. Left: Simulated Zn K-edge EXAFS transforms for bulk sphalerite and nanoparticle model structures: initial (unrelaxed) $3 \mathrm{~nm}$ sphalerite model nanoparticle; MD relaxed water-coated nanoparticle; and MD relaxed uncoated nanoparticle. The whole-nanoparticle EXAFS calculations included contributions from the first three shells only, with Debye Temperatures: $\Theta_{1}=430 \mathrm{~K}$, $\Theta_{2}=\Theta_{3}=260 \mathrm{~K}$. Inset: Simulated EXAFS transform for MD relaxed watercoated nanoparticle superimposed against experimental water-coated EXAFS transform.

Right: Experimental Zn K-edge XANES spectrum for water-coated ZnS nanoparticles (solid curve) compared with simulated XANES spectra for bulk sphalerite (top curve). Plus simulated whole-nanoparticle XANES spectra for model nanoparticles structures: initial (unrelaxed) $3 \mathrm{~nm}$ sphalerite model nanoparticle; and MD relaxed water-coated nanoparticle. The parameters for the XANES calculations are given in Table 2.

Figure 10. Theoretical calculations of WAXS and Zn K-edge EXAFS spectra for six spherical $2.5 \mathrm{~nm}$ model $\mathrm{ZnS}$ nanoparticles, subjected to random atomic displacements that add static disorder, until the sum of the mean squared displacements (relative to the initial perfect structure) reached $0.032 \pm 0.001 \AA^{2}$ in every case. For three model nanoparticles, the disorder was applied uniformly; for the remaining three it was applied non-uniformly (surface- 
weighted). In a), the Q-weighted WAXS spectra are indistinguishable, while in b), each $k^{3}$-weighted first-shell EXAFS spectrum is distinct. In particular, the amplitudes of the EXAFS oscillations are systematically lower for uniform vs. non-uniform disorder. In c) we plot the $Q^{3}$-weighted WAXS structure factor of the first-shell only, obtained by applying the inverse sine transform to the firstshell peak in the real-space radial distribution function associated with a). These spectra show a similar sensitivity to the distribution of disorder as the EXAFS spectra in b).

Table 1. Fit results from $\mathrm{Zn} \mathrm{K}$ edge EXAFS of ZnS bulk (sphalerite) and nanoparticles in methanol, after the addition of water, or dried. Fit ranges: $k=2.5-12 \AA^{-1} ; r=1.2-2.8 \AA . S_{o}^{2}=0.96$ was obtained from a fit to the sphalerite reference. Values in square brackets were held constant during the fit. Errors: $\partial(\mathrm{CN}) \approx 0.5 ; \partial(R) \approx 0.01 \AA ; \partial(\mathrm{MSRD}) \approx 1.6 \times 10^{-3} \AA^{2} ; \partial\left(C_{3}\right) \approx 2 \times 10^{-4} \AA^{2}$.

Table 2. Zn K-edge EXAFS parameters: the best fit values of Debye temperature, $\Theta_{\mathrm{D} 3}$, to the first three shells of sphalerite and wurtzite (fit given in Fig. 8), using the Hedin-Lundqvist exchange potential. Zn K-edge XANES parameters: $\Theta_{D}$ is the Debye temperature; $R_{s c f}\left(R_{f m s}\right)$ is the cluster radius for the self-consistent muffin-tin potential (full multiple scattering) calculation; $I_{\text {scf }}$ $\left(I_{\mathrm{fms}}\right)$ is the maximum angular momentum contribution for the self-consistent muffin-tin potential (full multiple scattering) calculation. $\Delta \mathrm{E}_{\mathrm{F}}$ is the Fermi energy shift. $\Delta \mathrm{E}_{\mathrm{i}}$ is a constant broadening contribution. We used the Dirac-Hara exchange potential plus the INTERSTITIAL card, as detailed in the FEFF manual [29]. 
